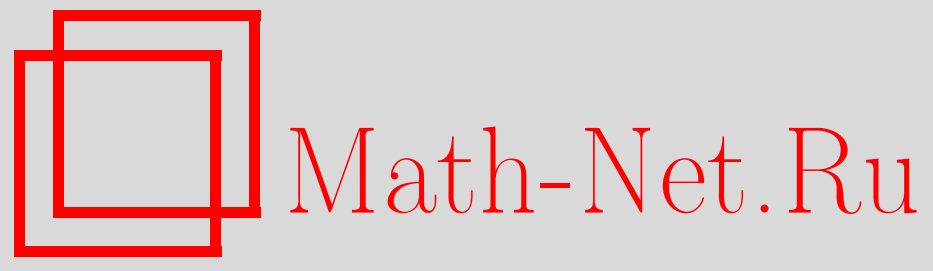

Ж. А. Балкизов, А. А. Сокуров, Об одном разностном методе решения задачи Трикоми для уравнения Лаврентьева-Бицадзе, Вестн. Сам. гос. техн. ун-та. Сер. Физ.-мат. науки, 2017, номер 2, 221235

DOI: https://doi.org/10.14498/vsgtu1534

Использование Общероссийского математического портала MathNet.Ru подразумевает, что вы прочитали и согласны с пользовательским соглашением

http: //www . mathnet.ru/rus/agreement

Параметры загрузки:

IP: 3.89 .185 .249

26 апреля 2023 г., $17: 53: 51$

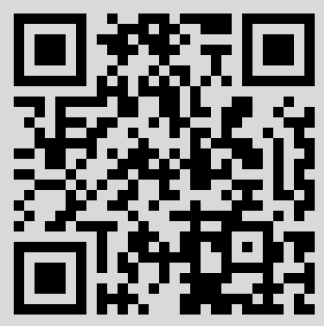


Вестн. Сам. гос. техн. ун-та. Сер. Физ.-мат. науки. 2017. Т. 21, № 2 . С. $221-235$ ISSN: 2310-7081 (online), 1991-8615 (print)

УДК 519.63

\title{
Об одном разностном методе решения задачи Трикоми для уравнения Лаврентьева-Бицадзе
}

\author{
Ж. А. Балкизов, А. А. Сокуров \\ Институт прикладной математики и автоматизации, \\ Россия, 360000, Нальчик, ул. Шортанова, 89 а.
}

\begin{abstract}
Аннотация
Получена априорная оценка решения задачи Трикоми для уравнения Лаврентьева-Бицадзе, из которой следует единственность регулярного решения. Предложен разностный метод нахождения приближенного решения исследуемой задачи. Получена априорная оценка решения разностной задачи, из которой следует сходимость построенной разностной схемы со вторым порядком точности.
\end{abstract}

Ключевые слова: уравнение смешанного типа, задача Трикоми, априорная оценка, разностная схема, порядок аппроксимации, метод энергетических неравенств.

Получение: 20 марта 2017 г. / Исправление: 7 мая 2017 г. /

Принятие: 12 июня 2017 г. / Публикация онлайн: 10 июля 2017 г.

Введение. Уравнение Лаврентьева-Бицадзе (ЛБ) в работах $[1,2]$ было предложено как более простая модель уравнений смешанного типа в смысле постановки и исследования смешанных задач. Методом сингулярных интегральных уравнений в работах [1-6] решена задача Трикоми для уравнения ЛБ для различных областей в эллиптической части. Исследованию однозначной разрешимости локальных и нелокальных краевых задач для уравнения ЛБ, а также его обобщениям посвящено достаточно много работ. Отметим, например, работы [7-13]. Однако, несмотря на обширную библиографию работ по исследованию однозначной разрешимости различных форм дифференциальных задач, приближенным методам исследования краевых задач для

\section{Статья}

ə () Контент публикуется на условиях лицензии Creative Commons Attribution 4.0 International (https://creativecommons.org/licenses/by/4.0/deed.ru)

\section{Образец для цитирования}

Бал кизов Ж. А., С оку ров А. А. Об одном разностном методе решения задачи Трикоми для уравнения Лаврентьева-Бицадзе // Вестн. Сам. гос. техн. ун-та. Сер. Физ.-мат. науки, 2017. Т. 21, № 2. C. 221-235. doi: 10.14498/vsgtu1534.

\section{Сведения об авторах}

Жираслан Анатолвевич Балкизов (1D http://orcid.org/0000-0001-5329-7766

кандидат физико-математических наук; заведующий отделом; отдел уравнений смешанного типа; e-mail: giraslan@yandex.ru

Аслан Артурович Сокуров (10) http://orcid.org/0000-0001-9886-3602

младший научный сотрудник; отдел теоретической и математической физики;

e-mail: asokuroff@gmail.com 
уравнений смешанного типа уделено мало внимания. Среди них отметим работы [14-19], в которых различными методами были получены приближенные решения задачи Трикоми для уравнения ЛБ с первым порядком аппроксимации. В данной работе получена априорная оценка решения задачи Трикоми для уравнения ЛБ в случае, когда область эллиптичности ограничена прямоугольником, а также предложен метод приближенного решения исследуемой задачи со вторым порядком точности. Отметим, что уравнение ЛБ встречается при решении таких важных вопросов прикладного характера, как задачи теории бесконечно малых изгибаний поверхностей вращения, задачи безмоментной теории оболочек и т. д. Значительную роль такие уравнения играют и в задачах газовой динамики [20].

1. Постановка задачи. На евклидовой плоскости независимых переменных $x$ и $t$ рассмотрим уравнение Лаврентьева-Бицадзе

$$
u_{x x}+\operatorname{sgn}(t) u_{t t}=-f(x, t)
$$

где $\operatorname{sgn}(t)$ - знак числа $t ; f(x, t)$ - заданная функция; $u=u(x, t)$ - искомая функция.

Через $\Omega$ обозначим область, ограниченную при $t<0$ характеристиками $A C: x+t=0$ и $C B: x-t=l$ уравнения (1), выходящими из точек $A=(0,0)$ и $B=(l, 0)$ и пересекающимися в точке $C=(l / 2,-l / 2)$, а при $t>0$ - прямоугольником с вершинами в точках $A, B, A_{0}=(0, T), B_{0}=(l, T)$, $l>0, T>0$. Верхнюю часть области $\Omega$ при $t>0$ обозначим через $\Omega^{+}$, а нижнюю часть при $t<0$ через $\Omega^{-}$.

Уравнение (1) является уравнением смешанного типа: оно эллиптично в области $\Omega^{+}$и гиперболично в $\Omega^{-}$.

Регулярным в области $\Omega$ решением уравнения (1) назовем всякую функцию $u=u(x, t)$ из класса $C(\bar{\Omega}) \cap C^{1}(\Omega) \cap C^{2}\left(\bar{\Omega}^{+}\right) \cap C^{2}\left(\Omega^{-}\right)$, при подстановке которой уравнение (1) обращается в тождество.

В работе рассматривается задача Трикоми для уравнения (1) в следующей постановке.

ЗАДАчА Т. Найти регулярное в области $\Omega$ решение $u=u(x, t)$ уравнения (1), удовлетворяющее краевым условиям

$$
\begin{gathered}
u(l, t)=u_{1}(t), \quad u(0, t)=u_{3}(t), \quad 0 \leqslant t \leqslant T \\
u(x, T)=u_{2}(x), \quad 0 \leqslant x \leqslant l \\
u(x,-x)=\psi(x), \quad 0 \leqslant x \leqslant l / 2
\end{gathered}
$$

где $u_{1}(t), u_{2}(x), u_{3}(t), \psi(x)$ - заданные достаточно гладкие функиии, для которых выполнены следующие условия согласования:

$$
u_{3}(0)=\psi(0), \quad u_{3}(T)=u_{2}(0), \quad u_{2}(l)=u_{1}(T) .
$$

В работах [14-19] были предложены различные методы нахождения приближенного решения задачи Т для однородного уравнения Лаврентьева-Бицадзе с первым порядком точности. В настоящей работе предлагается конечно-разностный метод, позволяющий найти приближенное решение задачи Т 
для неоднородного уравнения Лаврентьева-Бицадзе со вторым порядком точности.

В дальнейшем будем предполагать, что $f(x, t) \in C(\bar{\Omega})$ и решение задачи (1)-(4) существует. Решение задачи Коши для уравнения (1) в области $\bar{\Omega}-$ можно представить в виде

$$
u(x, t)=\frac{\tau(x+t)+\tau(x-t)}{2}+\frac{1}{2} \int_{x-t}^{x+t} \nu(\xi) d \xi+\frac{1}{2} \int_{0}^{t} \int_{x-t+\eta}^{x+t-\eta} f(\xi, \eta) d \xi d \eta
$$

где $\tau(x)=u(x, 0), \nu(x)=u_{t}(x, 0)$. Учитывая условие $(4)$, из (5) получим

$$
u(x,-x)=\frac{\tau(0)+\tau(2 x)}{2}+\frac{1}{2} \int_{2 x}^{0} \nu(\xi) d \xi+\frac{1}{2} \int_{0}^{-x} \int_{2 x+\eta}^{-\eta} f(\xi, \eta) d \xi d \eta=\psi(x)
$$

или же

$$
\frac{\tau(0)+\tau(x)}{2}+\frac{1}{2} \int_{x}^{0} \nu(\xi) d \xi+\frac{1}{2} \int_{0}^{-x / 2} \int_{x+\eta}^{-\eta} f(\xi, \eta) d \xi d \eta=\psi\left(\frac{x}{2}\right) .
$$

Дифференцируя (6), находим

$$
\tau^{\prime}(x)-\nu(x)=u_{x}(x, 0)-u_{t}(x, 0)=\psi^{\prime}\left(\frac{x}{2}\right)+\int_{0}^{-x / 2} f(x+\eta, \eta) d \eta,
$$

откуда

$$
\nu(x)=\tau^{\prime}(x)-\psi^{\prime}\left(\frac{x}{2}\right)-\int_{0}^{-x / 2} f(x+\eta, \eta) d \eta .
$$

Соотношение (8) - фундаментальное соотношение между функциями $\tau(x)$ и $\nu(x)$, принесенное из области $\Omega^{-}$на линию $y=0$. Подставляя $\nu(x)$ из $(8)$ в (5), находим решение первой задачи Дарбу для уравнения (1) в области $\Omega^{-}$:

$$
\begin{aligned}
u(x, t)=\tau(x+t) & -\psi\left(\frac{x+t}{2}\right)+\psi\left(\frac{x-t}{2}\right)- \\
& -\frac{1}{2} \int_{x-t}^{x+t} \int_{0}^{-\xi / 2} f(\xi+\eta, \eta) d \eta d \xi+\frac{1}{2} \int_{0}^{t} \int_{x-t+\eta}^{x+t-\eta} f(\xi, \eta) d \xi d \eta
\end{aligned}
$$

Для определения значения искомой функции $u$ в области $\Omega^{+}$с учетом найденного выше фундаментального соотношения приходим к задаче (2), (3) и (7) для уравнения (1).

2. Априорная оценка. Справедлива следующая теорема.

Теорема 1. Для любого регулярного решения задачи (1)-(3) и (7) с однородными условиями (2)-(3) имеет место априорная оценка

$$
\|u\|_{0}^{2} \leqslant M_{1}\|f\|_{0}^{2}+M_{2}\|\chi\|_{0}^{2},
$$

əəe

$$
\chi(x)=\psi^{\prime}\left(\frac{x}{2}\right)+\int_{0}^{-x / 2} f(x+\eta, \eta) d \eta
$$


$M_{1}, M_{2}-$ известные положстельные числа.

Дока з ат ельств о. Полагая условия (2), (3) однородными, умножим уравнение $(1)$ на $u(x, t)$ и проинтегрируем по области $\Omega^{+}$:

$$
\begin{aligned}
& \iint_{\Omega^{+}} u(x, t)\left[u_{x x}(x, t)+u_{t t}(x, t)\right] d x d t= \\
& =\int_{\Gamma}-u(x, t) u_{t}(x, t) d x+u(x, t) u_{x}(x, t) d t-\iint_{\Omega^{+}}\left[u_{x}^{2}(x, t)+u_{t}^{2}(x, t)\right] d x d t= \\
& =-\iint_{\Omega^{+}} u(x, t) f(x, t) d x d t,
\end{aligned}
$$

откуда

$$
\int_{0}^{l} u(x, 0) u_{t}(x, 0) d x+\left\|u_{x}\right\|_{0}^{2}+\left\|u_{t}\right\|_{0}^{2}=\iint_{\Omega^{+}} u(x, t) f(x, t) d x d t .
$$

Подставляя $u_{t}(x, 0)$ из $(7)$ в $(9)$, приходим к равенству

$$
-\int_{0}^{l} u(x, 0) \chi(x) d x+\left\|u_{x}\right\|_{0}^{2}+\left\|u_{t}\right\|_{0}^{2}=\iint_{\Omega^{+}} u(x, t) f(x, t) d x d t
$$

Пользуясь $\varepsilon$-неравенством, убеждаемся в справедливости неравенств

$$
\begin{aligned}
\iint_{\Omega^{+}} u(x, t) f(x, t) d x d t \leqslant \iint_{\Omega^{+}}\left[\varepsilon_{1} u^{2}(x, t)+\frac{1}{4 \varepsilon_{1}} f^{2}(x, t)\right] d x d t & = \\
& =\varepsilon_{1}\|u\|_{0}^{2}+\frac{1}{4 \varepsilon_{1}}\|f\|_{0}^{2}
\end{aligned}
$$

и

$$
\begin{aligned}
-\int_{0}^{l} u(x, 0) \chi(x) d x \geqslant \int_{0}^{l}\left[-\varepsilon_{2} u^{2}(x, 0)\right. & \left.-\frac{1}{4 \varepsilon_{2}} \chi^{2}(x)\right] d x= \\
& =-\varepsilon_{2} \int_{0}^{l} u^{2}(x, 0) d x-\frac{1}{4 \varepsilon_{2}}\|\chi\|_{0}^{2} .
\end{aligned}
$$

С учетом (11), (12) неравенство (10) перепишется в следующем виде:

$$
-\varepsilon_{2} \int_{0}^{l} u^{2}(x, 0) d x-\frac{1}{4 \varepsilon_{2}}\|\chi\|_{0}^{2}+\left\|u_{x}\right\|_{0}^{2}+\left\|u_{t}\right\|_{0}^{2} \leqslant \varepsilon_{1}\|u\|_{0}^{2}+\frac{1}{4 \varepsilon_{1}}\|f\|_{0}^{2} .
$$

Найдем теперь оценку для $\int_{0}^{l} u^{2}(x, 0) d x$. Воспользовавшись неравенством Коши-Буняковского с учетом условия (3) получаем

$$
\int_{0}^{l} u^{2}(x, 0) d x=\int_{0}^{l}\left(\int_{0}^{T} u_{t}(x, t) d t\right)^{2} d x \leqslant \int_{0}^{l}\left(T \int_{0}^{T} u_{t}^{2}(x, t) d t\right) d x=
$$




$$
=T \int_{0}^{l} \int_{0}^{T} u_{t}^{2}(x, t) d x d t=T \iint_{\Omega^{+}} u_{t}^{2}(x, t) d x d t=T\left\|u_{t}\right\|_{0}^{2} .
$$

С учетом (14) неравенство (13) перепишется в следующем виде:

$$
\left\|u_{x}\right\|_{0}^{2}+\left(1-\varepsilon_{2} T\right)\left\|u_{t}\right\|_{0}^{2} \leqslant \varepsilon_{1}\|u\|_{0}^{2}+\frac{1}{4 \varepsilon_{1}}\|f\|_{0}^{2}+\frac{1}{4 \varepsilon_{2}}\|\chi\|_{0}^{2} .
$$

Оценим далее $\left\|u_{x}\right\|_{0}^{2}$. Для этого заметим, что

$$
u^{2}(x, t)=\left(\int_{0}^{x} u_{s}(s, t) d s\right)^{2} \leqslant x \int_{0}^{x} u_{s}^{2}(s, t) d s \leqslant x \int_{0}^{l} u_{x}^{2}(x, t) d x .
$$

Проинтегрируем неравенство (16) сначала по $x$ от 0 до $l$, а затем по $t$ от 0 до $T$ :

$$
\begin{aligned}
\int_{0}^{l} u^{2}(x, t) d x \leqslant \frac{l^{2}}{2} \int_{0}^{l} u_{x}^{2}(x, t) d x & \\
\int_{0}^{T}\left(\int_{0}^{l} u^{2}(x, t) d x\right) d t & =\iint_{\Omega^{+}} u^{2}(x, t) d x d t \leqslant \\
& \leqslant \frac{l^{2}}{2} \int_{0}^{T}\left(\int_{0}^{l} u_{x}^{2}(x, t) d x\right) d t=\frac{l^{2}}{2} \iint_{\Omega^{+}} u_{x}^{2}(x, t) d x d t .
\end{aligned}
$$

Или окончательно

$$
\frac{2}{l^{2}}\|u\|_{0}^{2} \leqslant\left\|u_{x}\right\|_{0}^{2}
$$

Для оценки $\left\|u_{t}\right\|_{0}^{2}$ проинтегрируем неравенство

$$
u^{2}(x, t)=\left(-\int_{t}^{T} u_{s}(x, s) d s\right)^{2} \leqslant t \int_{0}^{T} u_{t}^{2}(x, t) d t
$$

по области $\Omega^{+}$и, рассуждая аналогично, найдем

$$
\frac{2}{T^{2}}\|u\|_{0}^{2} \leqslant\left\|u_{t}\right\|_{0}^{2}
$$

С учетом (17) и (18) из (15) получаем

$$
M\|u\|_{0}^{2} \leqslant \frac{1}{4 \varepsilon_{1}}\|f\|_{0}^{2}+\frac{1}{4 \varepsilon_{2}}\|\chi\|_{0}^{2},
$$

где

$$
M=\frac{2}{l^{2}}+\frac{2}{T^{2}}-\frac{2 \varepsilon_{2}}{T}-\varepsilon_{1} .
$$

В силу произвольности $\varepsilon_{1}$ и $\varepsilon_{2}$ число $M$ можно выбрать положительным. Тогда окончательно получим

$$
\|u\|_{0}^{2} \leqslant M_{1}\|f\|_{0}^{2}+M_{2}\|\chi\|_{0}^{2},
$$


где

$$
M_{1}=\frac{1}{4 \varepsilon_{1} M}, \quad M_{2}=\frac{1}{\varepsilon_{2} M} .
$$

Из априорной оценки (19) следует единственность регулярного решения исследуемой задачи.

3. Разностная схема. Для нахождения приближенного численного решения задачи (1)-(3), (7) для уравнения (1) в области $\bar{\Omega}^{+}$воспользуемся методом конечных разностей. В $\bar{\Omega}^{+}$введем сетку

$$
\bar{\omega}_{h_{x} h_{t}}=\bar{\omega}_{h_{x}} \times \bar{\omega}_{h_{t}},
$$

где $\bar{\omega}_{h_{x}}=\left\{x_{i}=i h_{x}, i=\overline{0, N_{x}}\right\}, \bar{\omega}_{h_{t}}=\left\{t_{j}=j h_{t}, j=\overline{0, N_{t}}\right\}, h_{x}=l / N_{x}$, $h_{t}=T / N_{t}, N_{x}$ и $N_{t}$ - натуральные числа.

Исходной дифференциальной задаче поставим в соответствие следующую разностную схему:

$$
\begin{gathered}
\left(y_{\bar{x} x}\right)_{i}^{j}+\left(y_{\bar{t} t}\right)_{i}^{j}=-\varphi_{i}^{j}, \quad i=\overline{1, N_{x}-1}, j=\overline{1, N_{t}-1}, \\
y_{N_{x}}^{j}=u_{1}\left(t_{j}\right), \quad y_{0}^{j}=u_{3}\left(t_{j}\right), \quad j=\overline{0, N_{t}} \\
y_{i}^{N_{t}}=u_{2}\left(x_{i}\right), \quad i=\overline{1, N_{x}-1}, \\
\left(y_{\grave{x}}\right)_{i}^{0}-\left(y_{t}\right)_{i}^{0}-\frac{h_{t}}{2}\left(y_{\bar{x} x}\right)_{i}^{0}=w_{i}, \quad i=\overline{1, N_{x}-1} .
\end{gathered}
$$

где

$$
\varphi_{i}^{j}=f\left(x_{i}, t_{j}\right), \quad w_{i}=\frac{h_{t}}{2} f\left(x_{i}, 0\right)+\psi^{\prime}\left(\frac{x_{i}}{2}\right)+\int_{0}^{-x_{i} / 2} f\left(x_{i}+\eta, \eta\right) d \eta .
$$

Введем следующие обозначения:

$\| y]\left.\right|_{0} ^{2}=\sum_{j=1}^{N_{t}} \sum_{i=1}^{N_{x}}\left(y_{i}^{j}\right)^{2} h_{t} h_{x}, \quad\|y\|_{C(\bar{\omega})}=\max _{x \in \bar{\omega}}|y(x)|, \quad(y, v)=\sum_{j=1}^{N_{t}-1} \sum_{i=1}^{N_{x}-1} y_{i}^{j} v_{i}^{j} h_{t} h_{x}$.

Теорема 2. Для любого решения $y(x), x \in \bar{\omega}_{h_{x} h_{t}}$, задачи (20)-(23) с однородными условиями (21)-(22) справедлива априорная оценка

$$
\left.\left.\| y]\left.\right|_{0} ^{2} \leqslant M_{3} \| w\right]\left.\right|_{0} ^{2}+M_{4} \| \varphi\right]\left.\right|_{0} ^{2}
$$

где $M_{3}, M_{4}$ - известные положительные числа.

Д о каз а т ель с т в о. Пользуясь методом энергетических неравенств [21, с. 110], умножим (20) скалярно на $y$ :

$$
\left(y, y_{\bar{x} x}\right)+\left(y, y_{\bar{t} t}\right)=-(y, \varphi) .
$$

Проведем следующие вычисления: 


$$
\begin{aligned}
& \left(y, y_{\bar{x} x}\right)=\sum_{j=1}^{N_{t}-1} \sum_{i=1}^{N_{x}-1}\left(y_{\bar{x} x}\right)_{i}^{j} y_{i}^{j} h_{t} h_{x}=\sum_{j=1}^{N_{t}-1} \sum_{i=1}^{N_{x}-1} \frac{\left(y_{\bar{x}}\right)_{i+1}^{j}-\left(y_{\bar{x}}\right)_{i}^{j}}{h_{x}} y_{i}^{j} h_{x} h_{t}= \\
& =\sum_{j=1}^{N_{t}-1} \sum_{i=1}^{N_{x}-1}\left[\left(y_{\bar{x}}\right)_{i+1}^{j} y_{i}^{j}-\left(y_{\bar{x}}\right)_{i}^{j} y_{i-1}^{j}-\left(y_{\bar{x}}\right)_{i}^{j} y_{i}^{j}+\left(y_{\bar{x}}\right)_{i}^{j} y_{i-1}^{j}\right] h_{t}= \\
& =\sum_{j=1}^{N_{t}-1} \sum_{i=1}^{N_{x}-1}\left[\left(y_{\bar{x}}\right)_{i+1}^{j} y_{i}^{j}-\left(y_{\bar{x}}\right)_{i}^{j} y_{i-1}^{j}\right] h_{t}-\sum_{j=1}^{N_{t}-1} \sum_{i=1}^{N_{x}-1} \frac{y_{i}^{j}-y_{i-1}^{j}}{h_{x}}\left(y_{\bar{x}}\right)_{i}^{j} h_{t} h_{x}= \\
& =\sum_{j=1}^{N_{t}-1}\left[\left(y_{\bar{x}}\right)_{N_{x}}^{j} y_{N_{x}-1}^{j}-\left(y_{\bar{x}}\right)_{1}^{j} y_{0}^{j}\right] h_{t}-\sum_{j=1}^{N_{t}-1} \sum_{i=1}^{N_{x}-1}\left[\left(y_{\bar{x}}\right)_{i}^{j}\right]^{2} h_{t} h_{x}= \\
& =-\sum_{j=1}^{N_{t}-1} \frac{y_{N_{x}}^{j}-y_{N_{x}-1}^{j}}{h_{x}}\left(y_{\bar{x}}\right)_{N_{x}}^{j} h_{x} h_{t}-\sum_{j=1}^{N_{t}-1} \sum_{i=1}^{N_{x}-1}\left[\left(y_{\bar{x}}\right)_{i}^{j}\right]^{2} h_{t} h_{x}= \\
& =-\sum_{j=1}^{N_{t}-1}\left[\left(y_{\bar{x}}\right)_{N_{x}}^{j}\right]^{2} h_{t} h_{x}-\sum_{j=1}^{N_{t}-1} \sum_{i=1}^{N_{x}-1}\left[\left(y_{\bar{x}}\right)_{i}^{j}\right]^{2} h_{t} h_{x}=-\sum_{j=1}^{N_{t}-1} \sum_{i=1}^{N_{x}}\left[\left(y_{\bar{x}}\right)_{i}^{j}\right]^{2} h_{t} h_{x}= \\
& =-\sum_{j=1}^{N_{t}-1} \sum_{i=1}^{N_{x}}\left[\left(y_{\bar{x}}\right)_{i}^{j}\right]^{2} h_{t} h_{x}-\sum_{i=1}^{N_{x}}\left[\left(y_{\bar{x}}\right)_{i}^{N_{t}}\right]^{2} h_{t} h_{x}= \\
& \left.=-\sum_{j=1}^{N_{t}} \sum_{i=1}^{N_{x}}\left[\left(y_{\bar{x}}\right)_{i}^{j}\right]^{2} h_{t} h_{x}=-\| y_{\bar{x}}\right]\left.\right|_{0} ^{2}
\end{aligned}
$$

$$
\begin{gathered}
\left(y, y_{\bar{t} t}\right)=\sum_{i=1}^{N_{x}-1} \sum_{j=1}^{N_{t}-1}\left(y_{\bar{t}}\right)_{i}^{j} y_{i}^{j} h_{t} h_{x}=\sum_{i=1}^{N_{x}-1} \sum_{j=1}^{N_{t}-1} \frac{\left(y_{\bar{t}}\right)_{i}^{j+1}-\left(y_{\bar{t}}\right)_{i}^{j}}{h_{t}} y_{i}^{j} h_{x} h_{t}= \\
=\sum_{i=1}^{N_{x}-1} \sum_{j=1}^{N_{t}-1}\left[\left(y_{\bar{t}}\right)_{i}^{j+1} y_{i}^{j}-\left(y_{\bar{t}}\right)_{i}^{j} y_{i}^{j-1}-\left(y_{\bar{t}}\right)_{i}^{j} y_{i}^{j}+\left(y_{\bar{t}}\right)_{i}^{j} y_{i}^{j-1}\right] h_{x}= \\
=\sum_{i=1}^{N_{x}-1} \sum_{j=1}^{N_{t}-1}\left[\left(y_{\bar{t}}\right)_{i}^{j+1} y_{i}^{j}-\left(y_{\bar{t}}\right)_{i}^{j} y_{i}^{j-1}\right] h_{x}-\sum_{i=1}^{N_{x}-1} \sum_{j=1}^{N_{t}-1} \frac{y_{i}^{j}-y_{i}^{j-1}}{h_{t}}\left(y_{\bar{t}}\right)_{i}^{j} h_{t} h_{x}= \\
=\sum_{i=1}^{N_{x}-1}\left[\left(y_{\bar{t}}\right)_{i}^{N_{t}} y_{i}^{N_{t}-1}-\left(y_{\bar{t}}\right)_{i}^{1} y_{i}^{0}\right] h_{x}-\sum_{i=1}^{N_{x}-1} \sum_{j=1}^{N_{t}-1}\left[\left(y_{\bar{t}}\right)_{i}^{j}\right]^{2} h_{t} h_{x}= \\
=\sum_{i=1}^{N_{x}-1} \frac{y_{i}^{N_{t}}-y_{i}^{N_{t}-1}}{h_{t}}\left(y_{\bar{t}}\right)_{i}^{N_{t}} h_{t} h_{x}-\sum_{i=1}^{N_{x}-1}\left(y_{\bar{t}}\right)_{i}^{1} y_{i}^{0} h_{x}-\sum_{i=1}^{N_{x}-1} \sum_{j=1}^{N_{t}-1}\left[\left(y_{\bar{t}}\right)_{i}^{j}\right]^{2} h_{t} h_{x}= \\
=-\sum_{i=1}^{N_{x}-1}\left[\left(y_{\bar{t}}\right)_{i}^{N_{t}}\right]^{2} h_{t} h_{x}-\sum_{i=1}^{N_{x}-1}\left(y_{t}\right)_{i}^{0} y_{i}^{0} h_{x}-\sum_{i=1}^{N_{x}-1} \sum_{j=1}^{N_{t}-1}\left[\left(y_{\bar{t}}\right)_{i}^{j}\right]^{2} h_{t} h_{x}=
\end{gathered}
$$




$$
\begin{gathered}
=-\sum_{i=1}^{N_{x}-1}\left(y_{t}\right)_{i}^{0} y_{i}^{0} h_{x}-\sum_{i=1}^{N_{x}-1} \sum_{j=1}^{N_{t}}\left[\left(y_{\bar{t}}\right)_{i}^{j}\right]^{2} h_{t} h_{x}= \\
=-\sum_{i=1}^{N_{x}-1}\left(y_{t}\right)_{i}^{0} y_{i}^{0} h_{x}-\sum_{i=1}^{N_{x}-1} \sum_{j=1}^{N_{t}}\left[\left(y_{\bar{t}}\right)_{i}^{j}\right]^{2} h_{t} h_{x}-\sum_{j=1}^{N_{t}}\left[\left(y_{\bar{t}}\right)_{N_{x}}^{j}\right]^{2} h_{t} h_{x}= \\
\left.=-\sum_{i=1}^{N_{x}-1}\left(y_{t}\right)_{i}^{0} y_{i}^{0} h_{x}-\sum_{i=1}^{N_{x}} \sum_{j=1}^{N_{t}}\left[\left(y_{\bar{t}}\right)_{i}^{j}\right]^{2} h_{t} h_{x}=-\sum_{i=1}^{N_{x}-1}\left(y_{t}\right)_{i}^{0} y_{i}^{0} h_{x}-\| y_{\bar{t}}\right]\left.\right|_{0} ^{2} .
\end{gathered}
$$

Таким образом,

$$
\left.\left.\| y_{\bar{x}}\right]\left.\right|_{0} ^{2}+\| y_{\bar{t}}\right]\left.\right|_{0} ^{2}+\sum_{i=1}^{N_{x}-1}\left(y_{t}\right)_{i}^{0} y_{i}^{0} h_{x}=(y, \varphi) .
$$

Выразим $\left(y_{t}\right)_{i}^{0}$ из $(23)$ и подставим в $(24)$. Тогда

$$
\left.\left.\| y_{\bar{x}}\right]\left.\right|_{0} ^{2}+\| y_{\bar{t}}\right]\left.\right|_{0} ^{2}+\sum_{i=1}^{N_{x}-1}\left[\left(y_{\check{x}}\right)_{i}^{0}-w_{i}-\frac{h_{t}}{2}\left(y_{\bar{x} x}\right)_{i}^{0}\right] y_{i}^{0} h_{x}=(y, \varphi) .
$$

Легко заметить, что

$$
\sum_{i=1}^{N_{x}-1}\left(y_{\grave{x}}\right)_{i}^{0} y_{i}^{0} h_{x}=y_{N_{x}}^{0} y_{N_{x}-1}^{0}-y_{0}^{0} y_{1}^{0}=0,
$$

$$
\begin{aligned}
& \frac{h_{t} h_{x}}{2} \sum_{i=1}^{N_{x}-1}\left(y_{\bar{x} x}\right)_{i}^{0} y_{i}^{0}=\frac{h_{t}}{2}\left(y_{N_{x}}^{0} y_{\bar{x}}^{0} N_{x}-y_{0}^{0} y_{\bar{x}}^{0}\right)-\frac{h_{t} h_{x}}{2} \sum_{i=1}^{N_{x}} y_{\bar{x} i}^{2}= \\
&=-\frac{h_{t} h_{x}}{2} \sum_{i=1}^{N_{x}} y_{\bar{x}}{ }^{2}{ }_{i} .
\end{aligned}
$$

С учетом (26), (27) из (25) получаем

$$
\left.\left.\| y_{\bar{x}}\right]\left.\right|_{0} ^{2}+\| y_{\bar{t}}\right]\left.\right|_{0} ^{2}+\frac{h_{t} h_{x}}{2} \sum_{i=1}^{N_{x}} y_{\bar{x}}^{2}{ }_{i}^{0}-\sum_{i=1}^{N_{x}-1} w_{i} y_{i}^{0} h_{x}=(y, \varphi),
$$

откуда

$$
\left.\left.\| y_{\bar{x}}\right]\left.\right|_{0} ^{2}+\| y_{\bar{t}}\right]\left.\right|_{0} ^{2}-h_{x} \sum_{i=1}^{N_{x}-1} w_{i} y_{i}^{0} \leqslant(y, \varphi) .
$$

Применим к третьему слагаемому слева в (28) $\varepsilon$-неравенство:

$$
h_{x} \sum_{i=1}^{N_{x}-1} w_{i} y_{i}^{0} \leqslant h_{x} \sum_{i=1}^{N_{x}}\left(\frac{1}{4 \varepsilon_{1}} w_{i}^{2}+\varepsilon_{1}{y_{i}^{0}}^{2}\right) .
$$


Тогда из (28) получим

$$
\begin{gathered}
\left.\left.\left.\left.\| y_{\bar{x}}\right]\left.\right|_{0} ^{2}+\| y_{\bar{t}}\right]\left.\right|_{0} ^{2}-\frac{1}{4 \varepsilon_{1}} \sum_{i=1}^{N_{x}} w_{i}^{2} h_{x}-\varepsilon_{1} \sum_{i=1}^{N_{x}} y_{i}^{0} h_{x} \leqslant \varepsilon_{2} \| y\right]\left.\right|_{0} ^{2}+\frac{1}{4 \varepsilon_{2}} \| \varphi\right]\left.\right|_{0} ^{2}, \\
\left.\left.\left.\left.\left.\| y_{\bar{x}}\right]\left.\right|_{0} ^{2}+\| y_{\bar{t}}\right]\left.\right|_{0} ^{2}-\varepsilon_{2} \| y\right]\left.\right|_{0} ^{2}-\varepsilon_{1} \sum_{i=1}^{N_{x}} y_{i}^{0^{2}} h_{x} \leqslant \frac{1}{4 \varepsilon_{1}} \| w\right]\left.\right|_{0} ^{2}+\frac{1}{4 \varepsilon_{2}} \| \varphi\right]\left.\right|_{0} ^{2}
\end{gathered}
$$

где $\| w]\left.\right|_{0} ^{2}=\sum_{i=1}^{N_{x}} w_{i}^{2} h_{x}$.

Оценим $\sum_{i=1}^{N_{x}} y_{i}^{0^{2}} h_{x}$. Для этого заметим, что

$$
y_{i}^{0}=-\sum_{j=1}^{N_{t}}\left(y_{\bar{t}}\right)_{i}^{j} h_{t}
$$

откуда

$$
\left(y_{i}^{0}\right)^{2}=\left(\sum_{j=1}^{N_{t}}\left(y_{\bar{t}}\right)_{i}^{j} h_{t}\right)^{2} \leqslant T \sum_{j=1}^{N_{t}}\left[\left(y_{\bar{t}}\right)_{i}^{j}\right]^{2} h_{t} .
$$

Суммируя обе части неравенства (30) по $i$ от $i=1$ до $i=N_{x}$, приходим к оценке

$$
\left.\sum_{i=1}^{N_{x}}\left(y_{i}^{0}\right)^{2} h_{x} \leqslant T \sum_{i=1}^{N_{x}} \sum_{j=1}^{N_{t}}\left[\left(y_{\bar{t}}\right)_{i}^{j}\right]^{2} h_{t} h_{x}=T \| y_{\bar{t}}\right]\left.\right|_{0} ^{2} .
$$

С учетом (31) неравенство (29) примет вид

$$
\left.\left.\left.\left.\left.\| y_{\bar{x}}\right]\left.\right|_{0} ^{2}+\left(1-\varepsilon_{1} T\right) \| y_{\bar{t}}\right]\left.\right|_{0} ^{2}-\varepsilon_{2} \| y\right]\left.\right|_{0} ^{2} \leqslant \frac{1}{4 \varepsilon_{1}} \| w\right]\left.\right|_{0} ^{2}+\frac{1}{4 \varepsilon_{2}} \| \varphi\right]\left.\right|_{0} ^{2} .
$$

Найдем теперь разностные аналоги неравенств (17) и (18). Имеем

$$
\begin{gathered}
\left(y_{i}^{j}\right)^{2}=\left(\sum_{k=1}^{i}\left(y_{\bar{x}}\right)_{k}^{j} h_{x}\right)^{2} \leqslant i h_{x} \sum_{k=1}^{i}\left[\left(y_{\bar{x}}\right)_{k}^{j}\right]^{2} h_{x} \leqslant i h_{x} \sum_{k=1}^{N_{x}}\left[\left(y_{\bar{x}}\right)_{k}^{j}\right]^{2} h_{x}, \\
\sum_{i=1}^{N_{x}}\left(y_{i}^{j}\right)^{2} h_{x} \leqslant\left(\sum_{k=1}^{N_{x}}\left[\left(y_{\bar{x}}\right)_{k}^{j}\right]^{2} h_{x}\right)\left(\sum_{i=1}^{N_{x}} i h_{x}^{2}\right)=\frac{l\left(l+h_{x}\right)}{2} \sum_{i=1}^{N_{x}}\left[\left(y_{\bar{x}}\right)_{i}^{j}\right]^{2} h_{x}, \\
\sum_{j=1}^{N_{t}} \sum_{i=1}^{N_{x}}\left(y_{i}^{j}\right)^{2} h_{t} h_{x} \leqslant \frac{l\left(l+h_{x}\right)}{2} \sum_{j=1}^{N_{t}} \sum_{i=1}^{N_{x}}\left[\left(y_{\bar{x}}\right)_{i}^{j}\right]^{2} h_{t} h_{x}, \\
\left.\left.\frac{2}{l\left(l+h_{x}\right)} \| y\right]\left.\right|_{0} ^{2} \leqslant \| y_{\bar{x}}\right]\left.\right|_{0} ^{2} .
\end{gathered}
$$

откуда

Аналогично получаем оценку

$$
\left.\left.\frac{2}{T^{2}} \| y\right]\left.\right|_{0} ^{2} \leqslant \| y_{\bar{t}}\right]\left.\right|_{0} ^{2}
$$


С учетом (33), (34) из (32) приходим к оценке вида

$$
\left.\left.M \| y]\left.\right|_{0} ^{2} \leqslant \frac{1}{4 \varepsilon_{1}} \| w\right]\left.\right|_{0} ^{2}+\frac{1}{4 \varepsilon_{2}} \| \varphi\right]\left.\right|_{0} ^{2},
$$

где

$$
M=\frac{2}{l\left(l+h_{x}\right)}+\frac{2}{T^{2}}-\frac{2 \varepsilon_{1}}{T}-\varepsilon_{2} .
$$

Выбирая значения постоянных $\varepsilon_{1}$ и $\varepsilon_{2}$ так, чтобы $M>0$, из (35) окончательно приходим к априорной оценке

$$
\left.\left.\| y]\left.\right|_{0} ^{2} \leqslant M_{3} \| w\right]\left.\right|_{0} ^{2}+M_{4} \| \varphi\right]\left.\right|_{0} ^{2}
$$

где

$$
M_{3}=\frac{1}{4 \varepsilon_{1} M}, \quad M_{4}=\frac{1}{4 \varepsilon_{2} M} .
$$

Из априорной оценки (36) следует существование и единственность решения разностной задачи (20)-(23).

\section{4. Порядок аппроксимации и сходимость разностной схемы. Пусть} $u$ - точное решение задачи $(1)-(3),(7)$ из класса $C^{4}\left(\bar{\Omega}^{+}\right)$(завышенного условия гладкости на точное решение $u$ будем требовать для получения 2-го порядка аппроксимации), а $y$-решение соответствующей разностной задачи (20)-(23). Тогда для погрешности $z=y-u$ получаем задачу

$$
\begin{gathered}
\left(z_{\bar{x} x}\right)_{i}^{j}+\left(z_{\bar{t} t}\right)_{i}^{j}=-\bar{\varphi}_{i}^{j}, \quad i=\overline{1, N_{x}-1}, j=\overline{1, N_{t}-1} \\
z_{N_{x}}^{j}=0, \quad z_{0}^{j}=0, \quad j=\overline{0, N_{t}} \\
z_{i}^{N_{t}}=0, \quad i=\overline{1, N_{x}-1}, \\
\left(z_{\dot{x}}\right)_{i}^{0}-\left(z_{t}\right)_{i}^{0}-\frac{h_{t}}{2}\left(z_{\bar{x} x}\right)_{i}^{0}=\bar{w}_{i}, \quad i=\overline{1, N_{x}-1}
\end{gathered}
$$

где

$$
\bar{\varphi}_{i}^{j}=\left(u_{\bar{x} x}\right)_{i}^{j}+\left(u_{\bar{t} t}\right)_{i}^{j}+\varphi_{i}^{j}, \quad \bar{w}_{i}=-\left(\left(u_{\dot{x}}\right)_{i}^{0}-\left(u_{t}\right)_{i}^{0}-\frac{h_{t}}{2}\left(u_{\bar{x} x}\right)_{i}^{0}-w_{i}\right) .
$$

Пользуясь формулой Тейлора, оценим порядок малости сеточных функций $\bar{\varphi}$ и $\bar{w}$. Для $\bar{\varphi}$ получаем

$$
\begin{aligned}
\bar{\varphi}_{i}^{j}=\left(u_{\bar{x} x}\right)_{i}^{j}+\left(u_{\bar{t} t}\right)_{i}^{j}+\varphi_{i}^{j}= & \left(\frac{\partial^{2} u}{\partial x^{2}}\left(x_{i}, t_{j}\right)+\frac{\partial^{2} u}{\partial t^{2}}\left(x_{i}, t_{j}\right)+f\left(x_{i}, t_{j}\right)\right)+ \\
& +\frac{h_{x}^{2}}{12} \frac{\partial^{4} u}{\partial x^{4}}\left(\xi_{i}, t_{j}\right)+\frac{h_{t}^{2}}{12} \frac{\partial^{4} u}{\partial t^{4}}\left(x_{i}, \tau_{j}\right)=O\left(h_{x}^{2}+h_{t}^{2}\right),
\end{aligned}
$$

где

$$
\xi_{i}=x_{i}+\theta_{1} h_{x}, \quad\left|\theta_{1}\right| \leqslant 1, \quad \tau_{j}=t_{j}+\theta_{2} h_{t}, \quad\left|\theta_{2}\right| \leqslant 1 .
$$

Далее найдем порядок аппроксимации граничного условия (7). Для этого воспользуемся следующими разложениями:

$$
\left(u_{\dot{x}}^{\circ}\right)_{i}^{0}=\frac{\partial u}{\partial x}\left(x_{i}, 0\right)+\frac{h_{x}^{2}}{6} \frac{\partial^{3} u}{\partial x^{3}}\left(\bar{\xi}_{i}, 0\right),
$$




$$
\begin{gathered}
\left(u_{t}\right)_{i}^{0}=\frac{\partial u}{\partial t}\left(x_{i}, 0\right)+\frac{h_{t}}{2} \frac{\partial^{2} u}{\partial t^{2}}\left(x_{i}, 0\right)+\frac{h_{t}^{2}}{6} \frac{\partial^{3} u}{\partial t^{3}}\left(x_{i}, \overline{\tau_{i}}\right) \\
\left(u_{\bar{x} x}\right)_{i}^{0}=\frac{\partial^{2} u}{\partial x^{2}}\left(x_{i}, 0\right)+\frac{h_{x}^{2}}{12} \frac{\partial^{4} u}{\partial x^{4}}\left(\overline{\bar{\xi}}_{i}, 0\right)
\end{gathered}
$$

где $\overline{\xi_{i}}, \overline{\bar{\xi}}_{i}$ - некоторые промежуточные точки из интервала $\left(x_{i}-h_{x}, x_{i}+h_{x}\right)$, a $\bar{\tau}_{i} \in\left(0, h_{t}\right)$. С учетом приведенных выше разложений из $(23)$ находим

$$
\begin{aligned}
\left(u_{\dot{x}}\right)_{i}^{0}- & \left(u_{t}\right)_{i}^{0}-\frac{h_{t}}{2}\left(u_{\bar{x} x}\right)_{i}^{0}-w_{i}= \\
= & \left(\frac{\partial u}{\partial x}\left(x_{i}, 0\right)-\frac{\partial u}{\partial t}\left(x_{i}, 0\right)-\psi^{\prime}\left(\frac{x_{i}}{2}\right)-\int_{0}^{-x_{i} / 2} f^{-}\left(x_{i}+\eta, \eta\right) d \eta\right)- \\
& \quad-\frac{h_{t}}{2}\left(\frac{\partial^{2} u}{\partial x^{2}}\left(x_{i}, 0\right)+\frac{\partial^{2} u}{\partial t^{2}}\left(x_{i}, 0\right)+f\left(x_{i}, 0\right)\right)+\frac{h_{x}^{2}}{6} \frac{\partial^{3} u}{\partial x^{3}}\left(\bar{\xi}_{i}, 0\right)- \\
& \quad-\frac{h_{t}^{2}}{6} \frac{\partial^{3} u}{\partial t^{3}}\left(x_{i}, \overline{\tau_{i}}\right)-\frac{h_{t} h_{x}^{2}}{12} \frac{\partial^{4} u}{\partial x^{4}}\left(\overline{\bar{\xi}}_{i}, 0\right)=O\left(h_{x}^{2}+h_{t}^{2}\right) .
\end{aligned}
$$

Таким образом,

$$
\bar{\varphi}=O\left(h_{x}^{2}+h_{t}^{2}\right), \quad \bar{w}=O\left(h_{x}^{2}+h_{t}^{2}\right)
$$

Для z справедлива априорная оценка (36), откуда и будет следовать сходимость разностной схемы со скоростью $O\left(h_{x}^{2}+h_{t}^{2}\right)$ в норме $\left.\| \cdot\right]\left.\right|_{0}$.

5. Численные расчеты. Численные расчеты с использованием разностной схемы (20)-(23) были проведены для тестовой задачи в случае, когда входные данные заданы следующим образом:

$$
\begin{gathered}
f(x, t)= \begin{cases}\left(-2+36 t^{2}\right) \cos (6 x)+\left(2-25 x^{2}\right) \sin (5 t), & t>0 \\
10 t-2 \cos (t) \cos (x) \cosh (t)-\cos (x) \sin (t) \operatorname{sh}(t), & t<0\end{cases} \\
u_{1}(t)=-t^{2} \cos (6)+\sin (5 t), \quad u_{2}(x)=-\cos (6 x)+x^{2} \sin (5) \\
u_{3}(t)=-t^{2}, \quad \psi(x)=-5 x^{3}+\cos (x) \sin (x) \operatorname{sh}(x), \\
l=1, \quad T=1 .
\end{gathered}
$$

Точное решение задачи

$$
u(x, t)= \begin{cases}x^{2} \sin (5 t)-t^{2} \cos (6 x), & t>0 \\ 5 x^{2} t+\sin (t) \operatorname{sh}(t) \cos (x), & t<0\end{cases}
$$

Порядок сходимости вычислялся согласно формуле

$$
\log _{h_{1} / h_{2}}\left(\Delta_{1} / \Delta_{2}\right)
$$

где $\Delta_{i}$ - норма погрешности, соответствующая шагу $h_{i}=h_{x}=h_{t}$.

В таблице приведены значения погрешности и вычисленный на их основе порядок сходимости в нормах $\| \cdot]\left.\right|_{0}$ и $\|\cdot\|_{C(\bar{\omega})}$. Как видно, сгущение сетки приводит к уменьшению погрешности и порядок сходимости стремится 
Погрешность и порядок сходимости в нормах $\| \cdot]\left.\right|_{0}$ и $\|\cdot\|_{C(\bar{\omega})}$

[Error estimation and rate of convergence of the $\| \cdot]\left.\right|_{0}$ and $\|\cdot\|_{C(\bar{\omega})}$ norms]

\begin{tabular}{c||c|c|c|c}
\hline$h_{x}=h_{t}$ & $\begin{array}{c}\text { Error estimation } \\
\text { of the } \| z]\left.\right|_{0} \text { norm }\end{array}$ & $\begin{array}{c}\text { Rate of convergence } \\
\text { of the } \| z]\left.\right|_{0} \text { norm }\end{array}$ & $\begin{array}{c}\text { Error estimation } \\
\text { of the }\|z\|_{C(\bar{\omega})} \text { norm }\end{array}$ & $\begin{array}{c}\text { Rate of convergence } \\
\text { of the }\|z\|_{C(\bar{\omega})} \text { norm }\end{array}$ \\
\hline \hline $1 / 20$ & 0.00172 & & 0.00373 & \\
$1 / 40$ & $4.26217 \cdot 10^{-4}$ & 2.01029 & $9.22934 \cdot 10^{-4}$ & 2.01678 \\
$1 / 80$ & $1.06206 \cdot 10^{-4}$ & 2.00472 & $2.30968 \cdot 10^{-4}$ & 1.99853 \\
$1 / 160$ & $2.65094 \cdot 10^{-5}$ & 2.00228 & $5.77445 \cdot 10^{-5}$ & 1.99993 \\
\hline
\end{tabular}

к двум. Эти выводы согласуются с результатами, полученными при исследовании свойств разностной схемы (20)-(23).

Конкурирующие интересы. Мы не имеем конкурирующих интересов.

Авторский вклад и ответственность. Все авторы принимали участие в разработке концепции статьи и в написании рукописи. Авторы несут полную ответственность за предоставление окончательной рукописи в печать. Окончательная версия рукописи была одобрена всеми авторами.

Финансирование. Исследование выполнялось без финансирования.

\section{Библиографический список}

1. Лаврентьев М. А., Бицадзе А. В. К проблеме уравнений смешанного типа // Докл. АН CCCP, 1950. Т. 70, № 3. С. 373-376.

2. Бицадзе А. В. Уравнения смешанного типа. М.: АН СССР, 1959. 164 с.

3. Чибрикова Л. И. Новый метод решения одной краевой задачи смешанного типа / Учен. зап. Казанск. ун-та, Т. 117. Казань: Изд-во Казанск. ун-та, 1957. С. 44-47.

4. Чибрикова Л. И. $\mathrm{K}$ решению краевой задачи Трикоми для уравнения $u_{x x}+\operatorname{sgn} y u_{y y}=0 /$ Учен. зап. Казанск. ун-та, Т. 117. Казань: Изд-во Казанск. ун-та, 1957. С. 48-51.

5. Лернер М. Е., Пулькин С. П. О единственности решений задач с условиями Франкля и Трикоми для общего уравнения Лаврентьева-Бицадзе // Дифферени. уравнения, 1966. T. 2, № 9. C. 1255-1263.

6. Крикунов Ю. М. К задаче Трикоми для уравнения Лаврентьева-Бицадзе // Изв. вузов. Матем., 1974. № 2. С. 76-81.

7. Моисеев Е. И. О задаче Трикоми для уравнения Лаврентьева-Бицадзе // Дифберени. уравнения, 1981. Т. 17, № 2. С. 325-338.

8. Казиев В. М. Задача Трикоми для нагруженного уравнения Лаврентьева-Бицадзе // Дифферени. уравнения, 1979. Т. 15, №1. С. 173-175.

9. Сабитов К. Б. О задаче Трикоми для уравнения Лаврентьева-Бицадзе со спектральным параметром // Дифберени. уравнения, 1986. Т. 22, №11. С. 1977-1984.

10. Солдатов А. П. Задачи типа Дирихле для уравнения Лаврентьева-Бицадзе // Дифферени. уравнения, 1994. Т. 30, №11. С. 2001-2009.

11. Репин О. А. Задача Трикоми для уравнения смешанного типа в области, эллиптическая часть которого - полуполоса // Дифференц. уравнения, 1996. Т. 32, № 4. С. 565-567.

12. Лернер М. Е., Репин О. А. Об одной задаче с двумя нелокальными краевыми условиями для уравнения смешанного типа // Сиб. матем. журн., 1999. Т. 40, №6. С. 1260-1275.

13. Нахушева 3. А. О задаче Трикоми для уравнения Лаврентьева-Бицадзе с нелокальным условием сопряжения // Дифферени. уравнения, 2005. Т. 41, № 10. С. 1426-1428.

14. Ладыженская О. А. Об одном способе приближённого решения задачи ЛаврентьеваБицадзе // УМН, 1954. Т. 9, № 4(62). С. 187-189.

15. Халилов 3. И. Решение задачи для уравнения смешанного типа методом сеток // Докл. АН АзCCP, 1953. Т. 9, № 4. С. 189-190. 
16. Карманов В. Г. Об одной граничной задаче для уравнения смешанного типа // Докл. AH CCCP, 1954. Т. 95, № 3. С. 439-442.

17. Филиппов А. Ф. О разностном методе решения задачи Трикоми // Изв. АН СССР. Сер. матем., 1957. Т. 21, № 1. С. 73-88.

18. Ogawa H. On Difference Methods for the Solution of a Tricomi Problem// Trans. Amer. Math. Soc., 1961. vol.100, no. 3. pp. 404-424. doi: 10.2307/1993522.

19. Сабитов К. Б., Мугафаров М. Ф. Экстремальные свойства решений разностной задачи Трикоми для одной сеточной системы уравнений смешанного типа и их применения // Изв. вузов. Матем., 2005. № 4. С. 56-69.

20. Франкль Ф. И. Два газодинамических приложения краевой задачи ЛаврентьеваБицадзе // Вестник ЛГУ. Сер. матем., мех. и астр., 1951. Т. 6, №11. С. 3-7.

21. Самарский А. А. Теория разностных схем. М.: Наука, 1983. 616 с. 
Vestn. Samar. Gos. Tekhn. Univ., Ser. Fiz.-Mat. Nauki

[J. Samara State Tech. Univ., Ser. Phys. Math. Sci.], 2017, vol. 21, no. 2, pp. 221-235

ISSN: 2310-7081 (online), 1991-8615 (print)

doi) http://doi.org/10.14498/vsgtu1534

MSC: 65M06, 65M12

\title{
Finite-difference method for solving Tricomi problem for the Lavrent'ev-Bitsadze equation
}

\author{
G. A. Balkizov, A. A. Sokurov \\ Institute of Applied Mathematics and Automation, \\ 89 a, Shortanov st., Nalchik, 360000, Russian Federation.
}

\begin{abstract}
In this paper we obtain an a priori estimate for solution of Tricomi problem for the Lavrent'ev-Bitsadze equation, from which the uniqueness of regular solution follows. Presented a numerical finite-difference method for solving the investigated problem. We obtain an a priori estimate for solution of the difference scheme, from which follows the second-order convergence.

Keywords: equation of mixed type, Tricomi problem, a priori estimate, difference scheme, order of approximation, method of energy inequalities.

Received: $20^{\text {th }}$ March, $2017 /$ Revised: $7^{\text {th }}$ May, $2017 /$

Accepted: $12^{\text {th }}$ June, $2017 /$ First online: $10^{\text {th }}$ July, 2017

Competing interests. We have no competing interests.

Authors' contributions and responsibilities. Each author has participated in the article concept development and in the manuscript writing. The authors are absolutely responsible for submitting the final manuscript in print. Each author has approved the
\end{abstract} final version of manuscript.

Funding. The research has not had any sponsorship.

\footnotetext{
Article

(ㅇ)(i) The content is published under the terms of the Creative Commons Attribution 4.0 International License (http://creativecommons.org/licenses/by/4.0/)

Please cite this article in press as:

Balkizov G. A., Sokurov A. A. Finite-difference method for solving Tricomi problem for the Lavrent'ev-Bitsadze equation, Vestn. Samar. Gos. Tekhn. Univ., Ser. Fiz.-Mat. Nauki [J. Samara State Tech. Univ., Ser. Phys. Math. Sci.], 2017, vol. 21, no. 2, pp. 221-235. doi: 10.14498/vsgtu1534 (In Russian).
}

Authors' Details:

Giraslan A. Balkizov (1) http://orcid.org/0000-0001-5329-7766

Cand. Phys. \& Math. Sci.; Head of Department; Dept. of Mixed Type Equations;

e-mail: giraslan@yandex.ru

Aslan A. Sokurov (D) http://orcid.org/0000-0001-9886-3602

Junior Research Scientist; Dept. of Theoretical and Mathematical Physics;

e-mail: asokuroff@gmail.com 


\section{References}

1. Lavrent'ev M. A., Bitsadze A. V. On the problem of equations of mixed type, Doklady Akad. Nauk SSSR, 1950, vol. 70, no. 3, pp. 373-376 (In Russian).

2. Bitsadze A. V. Uravneniia smeshannogo tipa [Equations of mixed type]. Moscow, USSR Academy of Sciences Publ., 1959, 164 pp. (In Russian)

3. Chibrikova L. I. New method of solving one boundary value problem of the mixed type, Uchenye Zapiski Kazanskogo Universiteta, 117. Kazan, Kazan State University, 1957, pp. 44-47 (In Russian).

4. Chibrikova L. I. To the solution of the Tricomi boundary value problem for equation $u_{x x}+\operatorname{sgn} y u_{y y}=0$, Uchenye Zapiski Kazanskogo Universiteta, 117. Kazan, Kazan State University, 1957, pp. 48-51 (In Russian).

5. Lerner M. E., Pul'kin S. P. Uniqueness of a solution of problems with Frankl' and Tricomi conditions for the general Lavrent'ev-Bitsadze equation, Differ. Uravn., 1966, vol. 2, no. 9, pp. 1255-1263 (In Russian).

6. Krikunov Yu. M. On the Tricomi problem for the Lavrent'ev-Bitsadze equation, Izv. Vyssh. Uchebn. Zaved. Mat., 1974, no. 2, pp. 76-81 (In Russian).

7. E. I. Moiseev The Tricomi problem for the Lavrent'ev-Bitsadze equation, Differ. Uravn., 1981, vol. 17, no. 2, pp. 325-338 (In Russian).

8. Kaziev V. M. The Tricomi problem for a loaded Lavrent'ev-Bitsadze equation, Differ. Uravn., 1979, vol. 15, no. 1, pp. 173-175 (In Russian).

9. Sabitov K. B. The Tricomi problem for the Lavrent'ev-Bitsadze equation with a spectral parameter, Differ. Uravn., 1986, vol. 22, no. 11, pp. 1977-1984 (In Russian).

10. Soldatov A. P. Dirichlet-type problems for the Lavrent'ev-Bitsadze equation, Differ. Equ., 1994, vol. 30, no. 11, pp. 1846-1853.

11. Repin O. A. The Tricomi problem for an equation of mixed type in a domain whose elliptic part is a half-strip, Differ. Equ., 1996, vol. 32, no. 4, pp. 571-574.

12. Lerner M. E., Repin O. A. A problem with two nonlocal boundary conditions for a mixed-type equation, Siberian Math. J., 1999, vol.40, no.6, pp. 1064-1078. doi: 10.1007/ BF02677530.

13. Nakhusheva Z. A. On the Tricomi Problem for the Lavrent'ev-Bitsadze Equation with a Nonlocal Transmission Condition, Differ. Equ., 2005, vol.41, no. 10, pp. 1505-1508. doi : 10. 1007/s10625-005-0307-y.

14. Ladyzhenskaya O. A. On a method of approximate solution of the Lavrent'ev-Bicadze problem, Uspekhi Mat. Nauk, 1954, vol. 9, no.4(62), pp. 187-189 (In Russian).

15. Khalilov Z. I. Solution of the problem for a mixed-type equation by the grid method, Doklady Akad. Nauk AzSSR, 1953, vol. 9, no. 4, pp. 189-190 (In Russian).

16. Karmanov V. G. On a boundary problem for an equation of mixed type, Doklady Akad. Nauk SSSR, 1954, vol. 95, no. 3, pp. 439-442 (In Russian).

17. Filippov A. F. On the application of the method of finite differences to the solution of the problem of Tricomi, Izv. Akad. Nauk SSSR Ser. Mat., 1957, vol.21, no.1, pp. 73-88 (In Russian).

18. Ogawa H. On Difference Methods for the Solution of a Tricomi Problem, Trans. Amer. Math. Soc., 1961, vol. 100, no. 3, pp. 404-424. doi : 10.2307/1993522.

19. Sabitov K. B., Mugafarov M. F. Extremal properties of solutions of the Tricomi difference problem for a difference system of equations of mixed type and their applications, Russian Math. (Iz. VUZ), 2005, vol.49, no. 4, pp. 53-66.

20. Frankl F. I. Two gas-dynamical applications of the Lavrentiev-Bitsadze boundary value problem, Vestnik Leningrad. Univ. Ser. Mat. Meh. Astronom, 1951, vol. 6, no. 11, pp. 3-7 (In Russian).

21. Samarskii A. A. Teoriia raznostnykh skhem [Theory of difference schemes]. Moscow, Nauka, 1983, 616 pp. (In Russian) 